\title{
Metabolic Profile and Cardiovascular Risk in a Population of Renal Transplant Recipients
}

\author{
M. Gonçalves*, P. Vieira, L. Resende, J. Durães, N. Rosa, J.A. Teixeira, and G. Silva \\ From the Department of Nephrology, Hospital Central do Funchal, Funchal, Portugal
}

\begin{abstract}
Introduction. Cardiovascular disease is more common in renal transplant recipients (RTRs) than in the general population, and is the major cause of both graft loss and patient death in RTRs.

Objectives. This study aimed to characterize the cardiovascular risk factors, calculate the 7-year risk for major adverse cardiac events and the 7-year risk for death in a population of RTRs using a cardiovascular risk calculator, and determine the main cardiovascular risk factors associated with increased prediction of major adverse cardiac event (MACE) and death.
\end{abstract}

Patients. This is a retrospective review of clinical data from 121 RTRs who are in followup programs at our institution, and who had a functioning and stable graft for longer than 6 months.

Results. Among 121 adult patients followed at our institution (59.5\% males, mean age of $49.6 \pm 13.8$ years, mean times for functioning grafts were $105 \pm 73.5 \mathrm{mo}$ ), $86.8 \%$ had hypertension, $19.8 \%$ had diabetes, $24.8 \%$ were current or former smokers, $61.9 \%$ had increased body mass index, and $71 \%$ had dyslipidemia. The 7-year risk for MACE was more than $10 \%$ in $38(31.4 \%)$ patients with age, diabetes, and smoke being independent risk predictors. The 7-year risk for death was more than $10 \%$ in $56(46.3 \%)$ patients with age, diabetes, blood pressure, smoking, and male gender being independent risk predictors.

Conclusion. There is a high prevalence of cardiovascular risk factors in a population of RTRs, and there is increased risk for MACE and death. Accurate risk prediction is important for physician decision support and patient education, promoting improved cardiovascular health of RTRs, and thus prolonging the survival of both patients and graft.

$\mathbf{C}$ ARDIOVASCULAR DISEASES (CVDs) are the leading cause of morbidity and mortality in patients with chronic renal disease [1,2]. Kidney transplantation, compared to dialysis, promotes the reduction of cardiovascular risk [2,3]. However, the mortality rates due to CVD in kidney transplant recipients (RTRs) are greater than in the general population $[2,4,5]$. The higher incidence of post-transplantation CVD is the result of an increase in the prevalence of traditional risk factors such as diabetes mellitus (DM), hypertension, cigarette smoking, and hyperlipidemia and accumulation of several nontraditional risk factors associated with CVD after kidney transplantation, such as acute rejection episodes and the use of immunosuppressive drugs [2,4-6].
Therefore, the risk-prediction equations defined and validated for the general population underestimate CVD risk for kidney transplant recipients because risk factors pertinent to kidney transplantation patients are not included in those equations [4,5].

Application of the CVD and mortality risk equations specifically tailored for RTRs [3], could help clinicians to

*Address correspondence to Miguel Gonçalves, Department of Nephrology, Hospital Central do Funchal, Avenida Luís de Camões $n^{\circ} 57$, 9004-514, Funchal, Portugal. E-mail: amvg. leugim@gmail.com

$0041-1345 / 15$ http://dx.doi.org/10.1016/j.transproceed.2015.03.031 
Table 1. Characteristics of the Study Population

\begin{tabular}{|c|c|}
\hline Variable & All Patients $(n=121)$ \\
\hline Recipient age (SD), y & $49.6 \pm 13.8$ \\
\hline Recipient sex: Men, n (\%)/Women, n (\%) & $72(59.5 \%) / 49(40.5 \%)$ \\
\hline Recipient race: white, $\mathrm{n}(\%) /$ black, $\mathrm{n}(\%)$ & $120(99.2 \%) / 1(0.8 \%)$ \\
\hline Time on dialysis, mo (SD) & $50.2 \pm 42$ \\
\hline $\begin{array}{l}\text { Dialysis modality before the transplantation: pre-emptive, } \\
\mathrm{n}(\%) / \text { hemodialysis, } \mathrm{n}(\%) / \text { peritoneal dialysis, } \mathrm{n}(\%)\end{array}$ & $7(5.8 \%) / 101(83.5 \%) / 13(10.7 \%)$ \\
\hline Number of transplants: first transplant, $\mathrm{n}(\%) /$ second or more, $\mathrm{n}(\%)$ & $116(95.9 \%) / 5(4.1 \%)$ \\
\hline Dialysis duration (SD), mo & $105.4 \pm 73.5$ \\
\hline Donor type: deceased, n (\%)/living, n (\%) & $107(88.4 \%) / 14(11.6 \%)$ \\
\hline Immunosuppressive regimen: TAC + MMF + PDN, $n(\%)$ & $53(43.8 \%)$ \\
\hline eGFR (SD), $\mathrm{mL} / \mathrm{min} / 1.73 \mathrm{~m}^{2}$ & $52.0 \pm 15.2$ \\
\hline $\begin{array}{l}\text { Primary cause of kidney failure: unknown, } \mathrm{n}(\%) / \text { chronic } \\
\text { glomerulopathy, } \mathrm{n}(\%) / \text { hypertension, } \mathrm{n}(\%) / \mathrm{ADPKD}, \mathrm{n}(\%) / \text { diabetes } \\
\text { mellitus, } \mathrm{n}(\%) / \text { other, } \mathrm{n}(\%)\end{array}$ & $41(33.9 \%) / 32(26.4 \%) / 11(9.1 \%) / 10(8.3 \%) / 6(5.0 \%) / 21$ (17.4\%) \\
\hline Lipid abnormalities & $86(71 \%)$ \\
\hline Total cholesterol (>5.18 mmol/L), $\mathrm{n}(\%)$ & $42(34.7 \%)$ \\
\hline LDL cholesterol (>2.59 mmol/L), $\mathrm{n}(\%)$ & $69(57 \%)$ \\
\hline Triglycerides (>1.67 mmol/L), $\mathrm{n}(\%)$ & $42(34.7 \%)$ \\
\hline HDL cholesterol: $o^{\dagger}<1.04 \mathrm{mmol} / \mathrm{L}, \mathrm{n}(\%) / q<1.17 \mathrm{mmol} / \mathrm{L}, \mathrm{n}(\%)$ & $7(5.8 \%) / 13(10.7 \%)$ \\
\hline BMI (SD) & $26.2 \pm 4.0$ \\
\hline $25 \leq \mathrm{BMl}<30 \mathrm{~kg} / \mathrm{m}^{2}, \mathrm{n}(\%)$ & $57(47.1 \%)$ \\
\hline $\mathrm{BMI} \geq 30 \mathrm{~kg} / \mathrm{m}^{2}, \mathrm{n}(\%)$ & $18(14.9 \%)$ \\
\hline Smoking status: current or former, $\mathrm{n}(\%)$ & $30(24.8 \%)$ \\
\hline
\end{tabular}

Abbreviations: TAC, tacrolimus; MMF, mycophenolate mofetil; PDN, prednisolone; eGFR, estimated glomerular filtration rate; ADPKD, autosomal dominant polycystic kidney disease; LDL, low-density lipoprotein; HDL, high-density lipoprotein; BMI, body mass index $\left(\mathrm{kg} / \mathrm{m}^{2}\right)$.

risk-stratify their patients, decision support, quality of care assessment, and patients education $[3,4]$.

The aims of this study were to characterize the cardiovascular risk factors, calculate the 7-year risk for major adverse cardiac events (MACE) and death, and determine the main cardiovascular risk factors associated with increased likelihood of MACE and death in an RTR population.

\section{PATIENTS AND METHODS}

Between November 2013 and January 2014, we collected detailed clinical data from 121 RTRs with a functioning and stable graft for longer than 6 months in follow-up programs at our institution.

Recipient data elements included age, gender, race, primary cause of kidney failure, time on dialysis, dialysis modality before transplantation, number of transplants, time functioning graft, smoking status (current, former, or never) and medical story (medication, baseline CVD, DM, hypertension). Donor data elements included donor (deceased or living). Follow-up data included immunosuppressive regimen, physical examination findings (anthropometric measures, systolic and diastolic blood pressure) and laboratory variables (serum creatinine and lipid values).

Race was defined as white, black, or other. Hypertension was defined by systolic blood pressure $\geq 130 \mathrm{~mm} \mathrm{Hg}$, diastolic $\geq 80 \mathrm{~mm} \mathrm{Hg}$ [7] or antihypertensive medication use. Diabetes was defined by the use of insulin or oral hypoglycemic medications or patient history. Reference values for total, low-density lipoprotein (LDL), and high-density lipoprotein (HDL) cholesterol levels and hypertriglyceridemia were based on the final report from the third report of the national cholesterol education program expert panel on detection, evaluation, and treatment of high blood cholesterol in adults [8]. Body mass index (BMI) was calculated using the formula: weight $(\mathrm{kg}) / \mathrm{height}(\mathrm{m})^{2}$. Classification is according to the cut-off points of the World Health Organization [9].
Estimated glomerular filtration rate (eGFR) was calculated using the four-variable Modification of Diet in Renal Disease Study equation, based on serum creatinine level, age, gender, and race [10]. MACE was defined as cardiac death, nonfatal myocardial infarction, or coronary revascularization procedure [3].

The formula for 7-year MACE and mortality risk calculation for RTRs [3] was used that is known as the Cardiovascular Risk Calculator for Renal Transplant Recipients (http://www.medsci. uu.se/forskning/Inflammation_och_autoimmunitet/Njurmedicin/ Projekt/risk-calculator/) [11].

Results were expressed as mean values \pm SD. Univariate analysis was performed using the $\chi^{2}$ test and Student $t$ test for categorical and continuous data, respectively. Variables were used in the logistic regression model to estimate odds ratio (OR) for higher rate $(>10 \%)$ of 7-year MACE and death. All $P$ values were two-tailed and a $P$ value $<.05$ was considered significant.

\section{RESULTS}

Among 121 adult patients followed at our institution (Table 1), 72 were males $(59.5 \%$ ) with a mean age of $49.6 \pm$ 13.8 years. Before transplantation, 101 patients $(83.5 \%)$ were on hemodialysis, 13 patients $(10.7 \%)$ were on peritoneal dialysis, and 7 patients $(5.8 \%)$ received a preemptive kidney transplant. The mean dialysis duration was $50.2 \pm 42$ months (minimum, 0 mo; maximum, $189 \mathrm{mo}$ ). The mean time of the functioning graft was 105.4.土.73.5 months (minimum, 6 mo; maximum, $358 \mathrm{mo}$ ). Recipients showed a mean eGFR of $52.0 \pm 15.2 \mathrm{~mL} / \mathrm{min} /$ $1.73 \mathrm{~m}^{2}$.

The causes of renal failure were glomerulonephritis $(26 \%)$, hypertension/nephrosclerosis $(9 \%)$, polycystic kidney 
Table 2. Univariate Analysis of Variables for 7-Year Risk for MACE*

\begin{tabular}{lccc}
\hline \multicolumn{1}{c}{ Variables } & Risk $<10 \%$ & Risk $\geq 10 \%$ & $P$ \\
\hline Age $(\mathrm{y})$ & $43.8 \pm 11.9$ & $62.4 \pm 7.4$ & .000 \\
Gender & & & \\
$\quad$ Male & $42(34.7 \%)$ & $30(24.8 \%)$ & .005 \\
$\quad$ Female & $41(33.9 \%)$ & $8(6.6 \%)$ & \\
Diabetes mellitus & $4(3.3 \%)$ & $20(16.5 \%)$ & .000 \\
Systolic blood pressure $(\mathrm{mm} \mathrm{Hg})$ & $127.2 \pm 14.9$ & $134.8 \pm 14.6$ & .01 \\
Current or former smoking & $13(10.7 \%)$ & $17(14.0 \%)$ & .001 \\
\hline
\end{tabular}

Abbreviations: MACE, major adverse cardiac events; CVD, cardiovascular disease; LDL, low-density lipoprotein; HDL, high-density lipoprotein.

*Dialysis duration, time functioning graft, baseline CVD, body mass index, estimated glomerular filtration rate, antihypertensive medications, total cholesterol level, LDL cholesterol, HDL cholesterol and triglycerides, showed no statistical significance.

disease (8\%), diabetic nephropathy (5\%), unknown (34\%), and other $(17 \%)$.

The prevalence of DM among our RTRs was $19.8 \%$. In this analysis, DM was a composite including type 1 and type $2 \mathrm{DM}$ diagnosed before transplantation and new onset DM after renal transplantation.

We found a high prevalence of hypertension among our patients, with $86.8 \%$ of patients who had high blood pressure or who received antihypertensive medication. The prevalence of lipid abnormalities among our study population was $71 \%$. In more detail, $34.7 \%$ of patients had high total cholesterol levels, $57 \%$ of patients had high LDL cholesterol levels, $16.5 \%$ had low HDL cholesterol levels (5.8\% of men and $10.7 \%$ of women), and there was hypertriglyceridemia in $34.7 \%$ of patients. Fifty-five percent of the patients had been treated with statin. The prevalence of cigarette smoking (current or former) among our patients was $24.8 \%$. High BMI was observed in $61.9 \%$ of patients, including $57(47.1 \%)$ who were overweight $\left(25 \leq \mathrm{BMI}<30 \mathrm{~kg} / \mathrm{m}^{2}\right)$ and $18(14.9 \%)$ who were obese $\left(\mathrm{BMI} \geq 30 \mathrm{~kg} / \mathrm{m}^{2}\right)$.

Using the Cardiovascular Risk Calculator for Renal Transplant Recipients, the 7-year risk for MACE was less than $10 \%$ in $83(68.6 \%)$ patients, between $10 \%$ and $20 \%$ in $22(18.2 \%)$ patients, and more than $20 \%$ in $16(13.2 \%)$ patients. The 7-year risk for death was less than $10 \%$ in 65 $(53.7 \%)$ patients, between $10 \%$ and $20 \%$ in $25(20.7 \%)$ patients, and more than $20 \%$ in $31(13.2 \%)$ patients.

Univariate analysis of variables for the 7-year risk for MACE (Table 2$)$ showed that older age $(P<.0001)$, male gender $(P=.005)$, DM $(P<.0001)$, higher systolic blood pressure $(P=.01)$, and current or former smoking $(P=$ $.001)$ were significant risk factors for the increased $(>10 \%)$ 7-year risk for MACE. The logistic regression model revealed that male gender $(\mathrm{OR}=8.14 ; P=.032 ; 95 \%$ confidence interval [CI], 1.193-55.546), age older than 55 years $(\mathrm{OR}=9.33 ; P=.001 ; 95 \% \mathrm{CI}, 2.439-35.717), \mathrm{DM}$ $(\mathrm{OR}=84.909 ; P<.0001 ; 95 \% \mathrm{CI}, 10.627-678.407)$, and current or former smoking $(\mathrm{OR}=9.481 ; P=.005 ; 95 \% \mathrm{CI}$, 1.996-45.047) were independent risk factors to predict a higher risk of MACE $(>10 \%)$. The same analysis confirmed a lack of correlation of dialysis duration, time of functioning graft, graft eGFR, BMI, number of antihypertensive drugs, and total cholesterol.

Regarding the 7-year risk for death (Table 3), older age $(P<.0001)$, male gender $(P=.002)$, DM $(P<.0001)$, higher systolic blood pressure $(P=.01)$, higher number of antihypertensive medications $(P=.041)$, current or former smoking $(P=.001)$, and higher total cholesterol level $(P=.002)$ were significant risk factors for the increased $(>10 \%)$ 7-year risk for death. The logistic regression model revealed that age older than 55 years $(\mathrm{OR}=$ 222.870; $P<.0001 ; 95 \%$ CI, 25.464-1950.608) and current or former smoking $(\mathrm{OR}=15.103 ; P=.004 ; 95 \% \mathrm{CI}$, 2.366-96.408) were independent risk factors to predict a higher risk of death $(>10 \%)$. The same analysis confirmed a lack of correlation of gender, dialysis duration, time of functioning graft, DM, graft eGFR, BMI, number of antihypertensive drugs, and total cholesterol.

\section{DISCUSSION}

RTRs are at increased risk of cardiovascular complications [2]. When compared with the general population, RTRs show a 4-fold greater risk for CVD and a 2-fold higher risk for death attributable to CVD [12].

Traditional risk factors for CVD, such as smoking, DM, and hypercholesterolemia, are well known to increase the risk of CVD [2]. In addition, there are specific transplantation risk factors such as deceased donors [13], longer duration of pretransplantation dialysis $[5,14]$, and immunosuppressive drugs [2,5,6]. Indeed, corticosteroids and calcineurin inhibitors have a range of potential effects on cardiovascular risk $[5,6]$, including increases in the risk and severity of hypertension, lipid abnormalities, and DM $[2,6]$.

In our study, we characterized the cardiovascular risk factors that were present in an RTR population. Our results show that the prevalence of DM among our population of transplant recipients was $19.8 \%$, lower than in other populations of renal transplantations [10], but much higher than the Framingham

Table 3. Univariate Analysis of Variables for 7-Year Risk for Death*

\begin{tabular}{lccc}
\hline \multicolumn{1}{c}{ Variables } & Risk $<10 \%$ & Risk $\geq 10 \%$ & $P$ \\
\hline Age $(\mathrm{y})$ & $39.8 \pm 10.3$ & $61.0 \pm 7.0$ & .000 \\
Gender & $30(\%)$ & $42(\%)$ & .002 \\
$\quad$ Male & $35(\%)$ & $14(\%)$ & \\
$\quad$ Female & $7(\%)$ & $17(\%)$ & .000 \\
Diabetes mellitus & $125.4 \pm 14.5$ & $134.5 \pm 14.5$ & .001 \\
Systolic blood pressure (mm Hg) & $8(10.7 \%)$ & $22(14.0 \%)$ & .001 \\
Current or former smoking & $1.6 \pm 1.1$ & $2.0 \pm 1.1$ & .041 \\
Antihypertensive medications & & & \\
$\quad$ (number) & & & \\
Total cholesterol level (mmol/L) & $4.59 \pm 0.94$ & $5.14 \pm 0.94$ & .002 \\
\hline
\end{tabular}

Abbreviations: CVD, cardiovascular disease; LDL, low-density lipoprotein; HDL, high-density lipoprotein.

*Dialysis duration, time functioning graft, baseline CVD, body mass index, estimated glomerular filtration rate, LDL cholesterol, HDL cholesterol and Triglycerides, showed no statistical significance. 
Heart Study population (5.2\% in males and $4.0 \%$ in females). The prevalence of hypertension varies between $21 \%$ and $26 \%$ among the general population, compared with $86.8 \%$ in our population of RTRs, overlapping with other existing studies in kidney transplantation (70\% to $85 \%$ ) [2]. Interestingly, the prevalence of lipid abnormalities among our RTRs was very high $(71 \%)$ compared with general population. However, the prevalence in our population overlaps with other populations of RTRs [2]. Despite the prevalence of lipid abnormalities, only $55 \%$ of patients are treated with statins. Comparing our results with other studies [2,5], it was evident that transplant recipients have several traditional risk factors (DM, hypertension, total cholesterol, LDL cholesterol, HDL cholesterol, triglycerides), with studies showing that these risk factors can predict the incidence of CVD [2,5].

Another aim of our study was to calculate the 7-year risk for MACE and death using a risk calculator specifically tailored for RTRs [3]. We use this calculator because, unlike calculators developed for the general population which do not include risk factors pertinent to RTRs, this prediction equation was drawn for assessment of cardiovascular risk and mortality risk among RTRs [3].

The prediction calculator $[3,11]$ showed that $31.4 \%$ and $13.2 \%$ of our population had an estimated probability of 7 -year MACE greater than $10 \%$ and $20 \%$, respectively. The mortality risk calculation in our population $[3,11]$ showed that $46.3 \%$ and $25.6 \%$ of our population had an estimated probability of 7-year death of more than $10 \%$ and $20 \%$, respectively. These results support the concept that RTRs have increased risk of cardiovascular complications and death. Application of the individualized and accurate prediction equations allows the clinical risk stratification of our patients.

Which factors most influence the increased risk CVD and death must be considered. In our study, we found a statistically significant relationship between increased cardiovascular risk and age, male gender, DM, systolic blood pressure, and current or former smoking. We also found a statistically significant relationship between the 7-year risk of death and age, male gender, DM, higher levels of systolic blood pressure, more antihypertensive medications, current or former smoking, and total cholesterol level. Our findings also complement the Cardiovascular Risk Calculator for Renal Transplant Recipients because new risk markers are constantly being proposed and are likely to be incorporated into risk prediction algorithms [3].

To our knowledge, this is the first application of the prediction of the 7-year risk of MACE and 7-year risk of death to an entire population of RTRs. More studies are essential for comparing risk between populations of RTRs and establish the desirable lower risk for these populations.

The application of this calculator individually for each patient works not only as an educational tool in motivating these patients, but also help physicians to stratify patients and to take appropriate measures to reduce individual cardiovascular risk or to diagnose and treat nonsymptomatic CVD, reducing the risk of MACE and death in RTRs.

\section{REFERENCES}

[1] Dimény EM. Cardiovascular disease after renal transplantation. Kidney Int Suppl 2002;61(80):78-84.

[2] Marcén R. Cardiovascular risk factors in renal transplantation - current controversies. Nephrol Dial Transplant 2006;21(Suppl 3):iii3-8.

[3] Soveri I, Holme I, Holdaas H, Budde K, Jardine AG, Fellström B. A cardiovascular risk calculator for renal transplant recipients. Transplantation 2012;94(1):57-62.

[4] Israni AK, Snyder JJ, Skeans MA, Peng Y, Maclean JR, Weinhandl ED, et al. Predicting coronary heart disease after kidney transplantation: Patient Outcomes in Renal Transplantation (PORT) Study. Am J Transplant 2010;10(2):338-53.

[5] Kasiske BL, Chakkera HA, Roel J. Explained and unexplained ischemic heart disease risk after renal transplantation. J Am Soc Nephrol 2000;11:1735-43.

[6] Fellström B. Risk factors for and management of posttransplantation cardiovascular disease. BioDrugs 2001;15(4): 261-78.

[7] Improving Global Outcomes (KDIGO) Blood Pressure Work Group. KDIGO Clinical Practice Guideline for the Management of Blood Pressure in Chronic Kidney Disease. Kidney Inter Suppl 2012;2:337-414.

[8] National Cholesterol Education Program (NCEP) Expert Panel on Detection, Evaluation, and Treatment of High Blood Cholesterol in Adults (Adult Treatment Panel III). Third Report of the National Cholesterol Education Program (NCEP) Expert Panel on Detection, Evaluation, and Treatment of High Blood Cholesterol in Adults (Adult Treatment Panel III) final report. Circulation 2002;106(25):3143-421.

[9] World Health Organization Expert Committee. Physical status: the use and interpretation of anthropometry. World Health Organ Tech Rep Ser 1995;854:1-452.

[10] Levey AS, Coresh J, Greene T, Stevens LA, Zhang YL, Hendriksen S, et al. Using standardized serum creatinine values in the modification of diet in renal disease study equation for estimating glomerular filtration rate. Ann Intern Med 2006;145(4): 247-54.

[11] Uppsala University [Internet]. Cardiovascular Risk Calculator for Renal Transplant Recipients, 2014. Available from: http:// www.medsci.uu.se/research/Inflammation + and+autoimmunity/ Renal + medicine/Projects/risk-calculator/?languageId=1. Accessed January 2014.

[12] Yazbek DC, de Carvalho AB, Barros CS, Marcassi AP, Pestana JO, Fachini FC, et al. Cardiovascular disease in early kidney transplantation: comparison between living and deceased donor recipients. Transplant Proc 2012;44(10):3001-6.

[13] Rigatto C, Parfrey P, Foley R, Negrijn C, Tribula C, Jeffery J. Congestive heart failure in renal transplant recipients: risk factors, outcomes, and relationship with ischemic heart disease. J Am Soc Nephrol 2002;13(4):1084-90.

[14] Abbott KC, Bucci JR, Cruess D, Taylor AJ, Agodoa LY. Graft loss and acute coronary syndromes after renal transplantation in the United States. J Am Soc Nephrol 2002;13(10): 2560-9. 